http://dx.doi.org/10.30681/23588403v14i0114

\title{
PRÁCTICAS DE LITERACIDAD DESIGUALES EN SOCIEDADES FRAGMENTADAS: LA HIDRA DEL CUENTO
}

\author{
María Antonieta Flores RAMOS (UFMT) ${ }^{1}$ \\ Data de recebimento: 04/05/2020 \\ Data de aceite: $26 / 07 / 2020$
}

\begin{abstract}
Resumo: A autora analisa as trajetórias desiguais de letramento de quatro profissionais com residência na cidade do México fazendo um comparativo dessas trajetórias com as trajetórias de letramento de indígenas maias estudantes no bacharelado onde Ramos trabalha como professora, em Chiapas, México. Ramos retoma a visão sociocultural e abrangente de Cassany e Castellá (2010) em torno à conceição de letramentos e, com o intuito de fazer um comparativo entre os processos de letramento, entrevista a cada uma dos quatro profissionais e posteriormente contrasta os depoimentos deles sobre o processo de alfabetização e aquisição dos letramentos com os testemunhos de sete estudantes maias reconhecendo assim que essas condutas adquiridas (Heath 2011) representam uma prática social onde interage a cultura herdada, o entorno político e educativo, e as crenças dos antecessores em torno ao aprendizado da leitura e a escrita. Logo da analise de um corpus de onze depoimentos a autora reconhece o fato de se tratar de uma pratica com trajetórias desiguais vistas as realidades socioculturais diversas em que os letramentos foram gestados. Os letramentos, compreendidos como práticas sócias e concepções sobre a leitura e escrita, geram um conjunto de reflexões que a autora remete a seu entorno imediato balizando-se em Street (1999), Cassany e Castellá (2010) e, primariamente, em Heath (2011).
\end{abstract}

Palavras-chave: Letramentos desiguais. Crenças sobre o uso das línguas. Espanhol-línguas indígenas.

\begin{abstract}
The author analyzes the unequal literacy trajectories of four professionals residing in Mexico City comparing these trajectories with the literacy trajectories of Mayan Indian students in the bachelor's degree where Ramos works as a teacher in Chiapas, Mexico. Ramos takes up the sociocultural and comprehensive view of Cassany and Castellá (2010) around the conception of literacies and, with the intention of comparing the literacy processes, interviews each of the four professionals and then contrasts their testimonies about the process of literacy with the testimonies of seven Mayan students, recognizing that these acquired conducts (Heath 2011) represent a social practice in which the inherited culture, the political and educational environment, and the predecessors' assumptions about learning reading and writing take place. After the analysis of a corpus of eleven testimonies, the author acknowledges the fact that literacy is a practice with uneven trajectories in view of the diverse sociocultural realities in which those practices were born. Literacy, understood as social practice and conception about, reading and writing, generate a set of reflections that the author refers to her immediate surroundings, based on Street (1999), Cassany and Castellá (2010) and, primarily, in Heath (2011).
\end{abstract}

Keywords: unequal literacy trajectories. Assumptions about languages. Spanish-indigenous languages.

\footnotetext{
${ }^{1}$ Investigación con el apoyo de la beca CAPES (código 001). Doctorante en el Programa de Posgrado en Estudios del Lenguaje de la Universidade Federal de Mato Grosso (PPGEL/UFMT). Profesora de base de la Universidad Intercultural de Chiapas, de nacionalidad mexicana, becaria de la OEA en convenio con GCUB (CAPES)
} 


\section{INTRODUCCIÓN}

La hidra de Lerna, personaje de la mitología griega, encarna, en términos generales, un problema de grandes dimensiones pues se trata de un animal que reproduce sus cabezas cuando se cree que todo ha cambiado para mejor, o bien, cuando la bestia se da por muerta al querer erradicarla de un tajo. Por eso Villanueva (2010) emplea esta metáfora, al hablar de la enseñanza de la lectoescritura en español, en un país fragmentado por las diferencias socioculturales y económicas donde el español se ha constituido como lengua imperial - y postcolonial- manifestándose en una serie de prácticas de literacidad que van desde el uso del español de "sobrevivencia" hasta el empleo erudito, bien pulido e inclusive bizarro de la llamada lengua de Cervantes.

Como ya lo han señalado Cassany e Castellá (2010), a semejanza de otros idiomas, las dificultades para referirse en español al término Literacy son conocidas y las opciones existentes poseen limitaciones, dadas las connotaciones que generan sin contar que ninguna de ellas se ha impuesto, unánimemente, en la comunidad académica. Por eso Cassany e Castellá (2010), analizan los términos en español correspondientes a literacy destacando las connotaciones asociadas a cada uno, las ventajas que cada cual conlleva, además de las desventajas de algunas formas que se emplean como sustantivos pero carecen de adjetivos, o viceversa. En suma, el termino literacidad empleado como préstamo de literacy incluye los términos traducidos como cultura escrita, escrituralidad o alfabetización y alfabetismo, entre otros (Cassany y Castellá, 2010). Este término, al cual nos adherimos en el presente ensayo, comprende una amplia gama de conocimientos, valores y actitudes asociados a la lectoescritura en una comunidad (Cassany e Castellá, 2010), además de incluir el dominio y uso:

del código alfabético, la construcción receptiva y productiva de textos, el conocimiento y el uso de las funciones y los propósitos de los diferentes géneros discursivos de cada ámbito social, los roles que adoptan el lector y el autor, los valores sociales asociados con estos roles (identidad, estatus, posición social), el conocimiento que se construye en estos textos y que circula en la comunidad (CASSANY; CASTELLÁ, 2010, p. 354)

\section{PARA LLEVAR A CABO ESTE ESTUDIO}

Para llevar a cabo este estudio entrevistamos a 11 personas -cuatro profesionistas residentes en la ciudad de México y siete estudiantes tsotsiles- las cuales, a pesar de diferencias 
de todo tipo, provienen de los dos estados mexicanos con mayor marginación y pobreza de la República Mexicana, sin contar, los más bajos índices de rezago educativo: Chiapas y Oaxaca. Siete de ellos son tsotsiles, uno zapoteco y, tres más, chiapanecos, procedentes de Cristóbal de Las Casas y residentes -los cuatro últimos- en la ciudad de México. Consecuentemente, se trata de cuatro profesionistas, de entre 47 y 50 años procedentes del estado de Oaxaca -el zapotecoy de Chiapas y, de 7 estudiantes tsotsiles cuya edad fluctúa entre los 20 y 24 años originarios de distintas regiones Chiapas, de los municipios de Huixtán, San Andrés Larrainzar, Aldana y Chenalhó salvo uno de ellos quien, a pesar de haber nacido y vivido toda su vida en San Cristóbal de Las Casas, se considera un tsotsil migrante a esta ciudad.

Todos ellos fueron entrevistados para preguntarles cómo se apropiaron de la lengua materna, cuáles fueron sus primeras experiencias con la lectoescritura, con las fuentes escritas y en qué momentos de convivencia intervenía el empleo de la lengua, o bien, la referencia a las fuentes escritas y, en el caso de los hablantes de una lengua originaria, cuáles son las estrategias empleadas para mantener viva su lengua. Los resultados fueron muy desiguales, pues los participantes poseen trayectorias de formación muy dispares relacionadas con su fase etaria, con las creencias en torno al empleo de las lenguas -por parte de la sociedad y de sus padres-, con el hecho de identificarse o carecer de ciertas prácticas de literacidad y con el apoyo -o bien la precariedad- de las instituciones escolares en enseñanza de la lengua; todo lo cual se vincula, en sentido general, con las prácticas sociales y culturales que rodearon a cada participante en su apropiación de la(s) lengua(s). Inspirada en el análisis de ciertas prácticas lingüísticas, espejo de sociedades fragmentadas en todo un amplio prisma económico y cultural del cual la lengua representa sólo un síntoma de un fenómeno mayor, decidí llevar a cabo una investigación comparativa cuyo nombre parafrasea el estudio llevado a cabo por Luchessi (2005).

Todos los participantes de la investigación fueron entrevistados con consentimiento informado y se les asigno nombres ficticios inspirados en la mitología griega que no delatan más que su género, algunos de estos nombres inclusive sugeridos por ellos mismos. Cabe mencionar que los tsotsiles -cuya lengua se refiere también a su grupo étnico- hablan, además del español, la lengua tsotsil una de las aproximadamente 30 lenguas de la familia lingüística maya, una de las 11 familias lingüísticas de México, hablada en Chiapas, México.

Las entrevistas se llevaron a cabo en el siguiente orden: primeramente entrevistamos a los participantes procedentes de San Cristóbal que residen en la ciudad de México; posteriormente, a los muchachos tsotsiles que estudian en San Cristóbal de Las Casas y, finalmente, al profesionista zapoteco residente en la ciudad de México. Todas las entrevistas, 
salvo la del profesionista zapoteco, se realizaron cara a cara con los participantes de la investigación; el profesionista zapoteco respondió mediante un video de 20 minutos las preguntas planteadas por la maestra Ramos. En lo sucesivo, plantearé algunas de los resultados derivados del primer grupo de entrevistas, es decir, del análisis aplicado a las hermanas profesionistas residentes en la ciudad de México; posteriormente, analizaré el caso del profesionista zapoteco -también residente en la ciudad de México- para finalmente contrastar sus testimonios con el de los estudiantes tsotsiles.

\section{LOS CUENTOS DE BUENAS NOCHES DE LAS HERMANAS PROFESIONISTAS}

Las hermanas profesionistas, en general, desarrollaron su formación en un entorno característico por la omnipresencia de las fuentes escritas, la valoración positiva de la lectura y la convivencia con personas de distintas edades, con intereses y valoraciones equivalentes. Desde edades tempranas, las hermanas tuvieron acceso a las prácticas de literacidad (CASSANY; CASTELLÁ, 2010) al interior de su familia, "primer y vigoroso entorno de aprendizaje" (MEEK, 2004, p.109) y no sólo sus padres fueron asiduos lectores, sino también un buen porcentaje de sus antepasados tanto de la familia materna como paterna. En términos generales, el objetivo de este primer grupo de entrevistas fue discernir, según HEATH (2011), en qué modelos de literacidad se encajaban las trayectorias de infancia y pre-adolescencia vividas por tres hermanas provincianas, radicadas en la ciudad de México. Si consideramos los tres modelos propuestos por HEATH (2011) en su ya clásico estudio sobre tres familias americanas, la apropiación de la lectoescritura y de la oralidad cuidada de las hermanas se sitúa entre el modelo Maintown y Roadville, aunque también posee características del modelo Trackton ¿Qué significa lo anterior? De manera muy esquemática significa lo siguiente:

En el caso de Maintown se trataba de niñas con tendencia a "desarrollar hábitos y valores que demostraban su adscripción a una sociedad letrada" (HEATH, 2011, p. 51-54). Vivían en entornos donde se gestaban ciertos rituales familiares con relación a las fuentes escritas no necesariamente de la lectura de cuentos sino de la lectura dominical de comics y otras revistas, dado que "existía fe en el poder y cualidades de la literacidad" (STREET, 1995, p.1), lo cual representa una práctica socialmente aprendida que manifiesta una ideología específica (STREET, 1995). Por su parte, los adultos alentaban las "platicas sobre libros" lo cual permitía "suspender la realidad" por momentos para darle paso a la ficción (HEATH, 2011). En su entorno, las actividades relacionadas con los libros $-\mathrm{u}$ otras fuentes impresas- se 
consideraban recreativas. Asimismo, en su conversación, muchas de sus referencias se basaban en fuentes escritas debido a la familiaridad con la lectura de libros.

En lo tocante al modelo Roadville, (HEATH, 2011), desde la infancia, las niñas participaban con hermanas, primos o niños mayores en juegos de ronda donde se requería "la anticipación y el reconocimiento de señales para futuros comportamientos" (HEATH, 2011 p. 66-70). Los padres de las hermanas, por su parte no siempre asumieron un rol tutorial en el aprendizaje pero brindaron las experiencias para esbozar el conocimiento.

\section{DE LA AUSENCIA A LA RECREACIÓN DE LOS TEXTOS}

Otro de los casos, el de un profesionista zapoteco residente hoy día en la ciudad de México, refleja una trayectoria de apropiación de las prácticas de literacidad muy interesante, pues estas han sido influidas por la valoración positiva del acto de leer y por la gratificación emotiva que Tiresias ha recibido como promotor de la lectura. Tiresias profesionista con posgrado y perteneciente al Sistema Nacional de Investigadores ha escrito artículos, capítulos de libros y libros sobre urbanismo e ingeniería. Es hijo de dos campesinos zapotecas procedentes de San Juan de Guelavía, Oaxaca que han vivido la mayor parte de sus vidas como indígenas urbanos residentes, primero, en Monterey y ahora en la ciudad de México. Tiresias narra que, en su infancia, no había más que dos libros en casa y que él no era muy allegado a las labores de limpieza; así, una vez que descubrió que su padre lo eximía de ellas cuando lo observaba leyendo, decidió refugiarse en la lectura, inicialmente para evitar las tareas domésticas, pero cada vez más por el regocijo y la apertura en el ángulo de mirada que los libros le otorgaban. Aunque Tiresias considera que las prácticas de literacidad representan una "capacidad cognitiva" -es decir, corresponden al modelo autónomo de literacidad según STREET (1995)- más desarrollada en unas personas que en otras no omite destacar que, desde su infancia, en su elenco de lecturas ya figuraban textos como Las mil y una noches, Heidi y El Principito, entre otros. En la Secundaria, es decir, en torno a los once y trece años (considerando que según su testimonio inició la educación básica a los cinco años), junto a dos amigos más, fue cautivado por las lecturas filosóficas de Friedrich Nietzsche. La lectura le permitió, desde temprana edad, distinguir entre un lector acrítico, que únicamente decodificaba letreros por cuestiones de la vida práctica, y un lector que manifestaba gozo, crítica y comentario en torno a lo que leía; en suma, gracias a ella, discernió, a decir de CASANY e CASTELLÁ (2010), la diferencia entre una lectura crítica y acrítica. 
En conjunto, la lectura crítica se sabe elaborada, compleja, humilde, cargada de matices y razonamientos, dúctil, insatisfecha, exploradora, capaz de discutir e, incluso, de cambiar de opinión de forma razonada; es la lectura que aspira a comprender la complejidad de lo humano, psicológico y social, y a transformarlo si es preciso. Al contrario, la acrítica se nos representa como sencilla, rápida, directa, simplificadora, autosatisfecha, esquemática y muchos otros adjetivos - no hay duda de que muchas de las características que aquí atribuimos a uno y otro tipo de lector tienen implicaciones didácticas de gran envergadura (CASSANY; CASTELLÁ, 2010, p. 367)

Este profesionista, además de detentor de una visión crítica de la lectoescritura con frecuencia refleja un vocabulario inspirado en las fuentes escritas (Vide modelo Maintown según HEATH, 2011) y manifiesta un repertorio, que, a veces, se antoja bizarro, de cultismos léxicos y de algunos latinismos. Debido a las actitudes encontradas de sus padres en relación al par de lenguas (y culturas) español-zapoteco, Tiresias conoció, en la infancia, precariamente el zapoteco. Tiresias narra que sus padres fueron alfabetizados en español de manera violenta por maestros rurales que llegaron a San Juan Guelavía, (Oaxaca, México) en los años cincuenta del siglo XX, y este aprendizaje atormentado del español sólo sucedió siendo adultos. De esta manera, las políticas educativas asimilacionistas encarnadas en el Vasconcelismo de los años cincuenta y las creencias en torno al prestigio de las lenguas -y de sus hablantes-, suplantaron al zapoteco, la lengua materna de sus padres, no sólo en su familia sino en este poblado, perteneciente al Valle de Tlacolula, en Oaxaca.

\section{LOS ESTUDIANTES TSOTSILES: ENTRE EXPERIENCIAS PROLÍFICAS Y LECTURAS PRECARIAS}

Los estudiantes tsotsiles, por su parte, ingresaron a la universidad en 2016, 2017 y 2018. El Catalogo de lenguas Indígenas del Instituto Nacional de Lenguas Indígenas o INALI establece siete variantes geolectales de la lengua tsotsil. Por su parte, el INALI como organismo descentralizado de la Administración Pública Federal mexicana procura responder a las disposiciones de la Ley General de Derechos Lingüísticos de los Pueblos Indígenas promoviendo el fortalecimiento, preservación y desarrollo de las lenguas indígenas habladas en el territorio mexicano. Al INALI correspondió, en el 2008, la elaboración de una catálogo de las lenguas indígenas mexicanas el cual presenta los nombres de las lenguas, las denominaciones establecidas por los propios hablantes y las variantes de cada agrupamiento linguíístico además de su ubicación geográfica entre otros datos. Según dicho Catalogo, las 
variantes de tsotsil habladas por los estudiantes tsotsiles son las siguientes: los dos hermanos de San Andrés Larrainzar hablan el tsotsil del Norte alto; otro más, ha vivido siempre en San Cristobal de Las Casas pero se adscribe a la comunidad de Yabteclum, municipio de Chenalhó correspondiente, al tsotsil del noroeste. Uno más habla el tsotsil de Los Altos pues procede de Pinar, municipio de San Cristóbal de Las Casas. Dos más, según el mismo Catalogo, hablan el tsotsil del este alto, pues radican en Adolfo López Mateos e Ispuilho, en el municipio de Huixtán y, el último, radica en San José Fiu, municipio de Aldama correspondiente al tsotsil del noreste.

En términos generales, sus estrategias de apropiación de la lengua tsotsil se basan en la convivencia con padres, primos, hermanos mayores -o menores- $\mathrm{y}$, en buena medida con abuelos quienes representan, para la mayoría de ellos, la medida de lo que significa hablar un buen tsotsil. Suelen proceder de familias numerosas donde las estrategias de aprendizaje se basan en la observación, pues una vez que cuentan con la madurez suficiente se les alienta a participar con los hermanos y los niños mayores de la comunidad en distintos juegos (como el escondedor o nutsbail) mediante los cuales se van apropiando de la lengua originaria (modelo Trackton, según HEATH 2011). También narran cómo en la participación de ciertos juegoscomo el ensamblaje de un carrito- intervienen los adultos brindando sus experiencias para gestionar el conocimiento de los niños (HEATH, 2011) y en este caso la imitación y observación juegan un papel primordial. Todos rememoran la convivencia con los abuelos, monolingües en tsotsil, como personas fecundas al recrear un contexto y "convocar a la audiencia a la hora de unirse en la creación imaginativa de una historia" (HEATH, 2011, p. 69). Uno de ellos menciona que fue enviado por sus padres a un internado a San Cristóbal de Las Casas con el objetivo de que aprendiera la lengua española pues ello representaría mayores oportunidades de empleo. Es decir existe la creencia de un beneficio derivado del aprendizaje del español, sin embargo este aprendizaje se relaciona, en realidad, con la apropiación de la oralidad y no necesariamente de la escritura.

Los estudiantes consideran que el aprendizaje efectivo de la lengua tsotsil se debe, más que nada, a la enseñanza de sus padres pues en las escuelas, aún las nombradas bilingües, la mayoría de las clases se impartían en español y el conocimiento de la escritura en tsotsil, en tres de los casos, se relaciona, primordialmente, con el aprendizaje del alfabeto como tal, la correspondencia entre sonido y grafía y, el aprendizaje de los números, el cual raras veces rebasa el número 20. En relación a lo anterior, una de las aportaciones del llamado Modelo educativo Intercultural, en este caso, es el (re) aprendizaje de la lectoescritura en lenguas 
originarias más allá del conocimiento de los números y letras, pues los estudiantes mencionan que en los trabajos académicos deben incluir resúmenes iniciales en tsotsil, es decir, debe haber, por lo menos, un ejercicio de elaboración de enunciados más allá del conocimiento del abecedario; en este mismo sentido, algunos de los maestros se aventuran también a asignarles tareas como la redacción de textos literarios breves a fin de analizar en clase asuntos diversos como el valor metafórico de las palabras, la manera convencional -y todavía no exenta de polémicas- de conformarlas según sus categorías gramaticales además de tocar la ortografía normalizada. En de mayo de 2019 con el consentimiento libre e informado de uno de los maestros de tsotsil fui invitada a participar en una clase de creación literaria en la cual se llevó a cabo el ejercicio descrito arriba. Se trataba de una clase impartida en tsotsil en la cual los muchachos analizaron un texto literario breve, elaborado previamente por ellos mismos.

En dos, de los siete casos, los estudiantes mencionaron que aprendieron la escritura en lengua española, en la escuela Primaria, gracias al valioso y paciente apoyo de sus maestros. La escuela Primaria, por su parte, representa el primer período de la educación básica más o menos correspondiente al Ensino Fundamental en Brasil la cual abarca seis grados de estudio -cursados en 6 años- e inicia cuando los niños cumplen seis años de edad y concluye cuando los niños tienen aproximadamente 12 años. En el primer caso de transmisión al estudiante de la lengua adicional por parte del profesor, el maestro rural hablaba tsotsil de Chamula e implementó los cursos de primero a cuarto de Primaria. Chamula es un municipio ubicado en la región Altos de Chiapas, México donde según el Catálogo del Instituto Nacional de Lenguas Indígenas se habla primordialmente tsotsil del centro pero que también cuenta con comunidades donde se habla tseltal de occidente. El conocimiento del profesor de ambas lenguas (españoltsotsil) le permitió la transmisión de una segunda lengua a los niños. En otro de los casos, el maestro hablante del tseltal de occidente, aprendió tsotsil para comunicarse con los estudiantes y así les transmitió el español a los niños tsotsiles. Según testimonio del muchacho entrevistado, su maestro de Primaria procedía del municipio de Oxchuc, Chiapas, es decir, según el Catalogo de INALI, hablaba tseltal de occidente. Por su parte, el tseltal, según la clasificación de idiomas de la familia lingüística maya hecha por Kauffmann (1974 apud ENGLAND, 1996, p. 9) es el idioma tipológicamente más próximo al tsotsil -separado de este hace aproximadamente 1400 años- que se desprende de la rama ch’ol, misma que a su vez pertenece a la División Occidental de la familia lingüística maya.

Estos casos de aprendizaje de la lectoescritura en L1 de manera menos aventurada, sin embargo, representan más bien esfuerzos aislados relacionados con la tenacidad individual 
-de maestro/a y alumno/a- más que con la eficiencia formativa del sistema educativo, sea bilingüe, o no. En el caso del aprendizaje de una L2 nos referimos, sin embargo, a la transmisión del castellano oral más que a las prácticas de literacidad en su conjunto pues hay muchos "ruidos" que afectan la comunicación escrita y la lectura crítica, tal como la conciben CASSANY y CASTELLÁ (2010).

Los estudiantes tsotsiles pertenecientes a la era de las fuentes multimodales y la multiliteracidad emplean los soportes electrónicos para mantener viva su lengua. Así, uno de ellos mencionó que en la página de facebook llamada "Chamula, tierra sagrada" reactualiza su conocimiento del tsotsil pues allí aparecen memes y textos breves en tsotsil retroalimentados por los propios hablantes. En general, su mayor referencia a las fuentes escritas durante la infancia es el Libro de Texto Gratuito (tanto en español como en tsotsil o batsil k'op) otorgados por la Secretaría de Educación Pública. El Libro de Texto Gratuito o LTG, más o menos equivalente al Livro Didático en Brasil fue creado en México en 1959 por decreto presidencial:

para encargarse de fijar, con apego a la metodología y a los programas respectivos, las características de los libros de texto destinados a la educación primaria cumpliendo así el mandato constitucional de proporcionar a los mexicanos una educación obligatoria y gratuita. La tríada gratuito-únicoobligatorio caracteriza hasta hoy al LTG el cual se distribuye a niños de escuelas primarias públicas y privadas, rurales y urbanas e inclusive posee versiones en 40 lenguas originarias. (ALEJOS, 2013 p.1196)

Sobre estos libros, reconocen algunas lecturas, además del tamaño y el color de los textos; raras veces aluden a nombres de autores. Dada su edad, las prácticas de literacidad más recientes (durante la educación media superior y la universidad, es decir a partir de los 14 años) se relacionan con los soportes electrónicos, pues allí leen los textos asignados como tareas, además de algunos comics o animés, principalmente, japoneses.

Los estudiantes tsotsiles advierten el valor de la lengua inglesa como hiperlengua que gana continuamente más espacio; sin embargo a la pregunta sobre qué lengua les gustaría transmitirle a sus hijos (A) y cuál aprenderían ellos si tuviera la oportunidad de hacerlo (B) respondieron lo siguiente: todos reconocieron que les gustaría enseñarle a sus hijos la lengua de sus abuelos (el tsotsil), pues el español, según sus consideraciones, lo aprenderían gracias al entorno. A la segunda pregunta, cinco de los siete, respondieron que les gustaría aprender el inglés dado a que lo asocian con mayores oportunidades de trabajo; uno de ellos respondió que le gustaría aprender el ch’ol o alguna otra lengua originaria de Chiapas. Uno más respondió que le gustaría aprender el japonés dada su afición a los animés japoneses. Entre los que 
respondieron que les gustaría aprender inglés, uno de ellos afirmó que le gustaría aprender chino dado que le encantaban los símbolos chinos.

\section{CONCLUSIONES INICIALES EN TORNO A LA HIDRA}

Como lo mencioné al inicio, en un país fragmentado por las diferencias sociales, culturales y económicas, las prácticas de literacidad manifiestan resultados variopintos; así, hablar de la lectoescritura puede significar experiencias desiguales -e inclusive opuestas- para los dos grupos analizados (los jóvenes tsotsiles y los profesionistas urbanos)

La literacidad tiene usos versátiles entre los profesionistas urbanos, pues se trata de prácticas permanentes asociadas a sus actividades del día a día, pero también inherentes a sus actividades profesionales. Los profesionistas urbanos migraron, de manera gradual, a los soportes electrónicos, pues nacieron en una etapa en que el boom de las Tecnologías de la Información y Comunicación no hacía acto de presencia, además de que la provincia mexicana era todavía poco industrializada. Pertenecen, pues, a una generación migrante a las fuentes multimodales de información que adaptaron sus prácticas de literacidad a las prácticas de multiliteracidad. A través de las redes sociales difunden y describen, brevemente, textos con los cuales se identifican, o bien, lecturas evaluadas como importantes para entender un tema.

Los jóvenes tsotsiles, por su parte, nacieron ya dentro del auge de las tecnologías de la Información y de la comunicación y todas las tareas realizadas en la escuela se relacionan con algún tipo de soporte electrónico; estos les permiten evitar gastos en fotocopias, libros o formatos diversos, Su relación con la literacidad se asocia con instituciones, principalmente con la escuela. A su vez, su relación con la lengua dominante tiene que ver principalmente con la apropiación de la lengua oral; en este sentido, no pertenecen a una cultura logocéntrica, pues la escritura posee valor en la medida en que resulta utilitaria en la vida práctica y se asocia también con actividades escolares, no siempre placenteras cuando se trata de escribir "textos largos". En general un texto largo no tiene que ver, necesariamente, con el tamaño del texto sino que se relaciona con un texto evaluable por las instituciones educativas, - merecedor de una calificación que, a su vez, genere consecuencias de distinto tipo- el cual deberá cubrir ciertas exigencias como evitar errores ortográficos groseros y cumplir con ciertos requisitos académicos, a veces poco claros. La mayoría de los estudiantes trabajan en la milpa durante el periodo de siembra, oportunidad para reactualizar sus conocimientos del tsotsil y fortalecer sus vínculos familiares. No existe entre ellos una estigmatización de sus antepasados no 
alfabetizados; contrariamente, los abuelos representan la mayor fuente de conocimiento en lo concerniente a la lengua y cultura originaria. La complacencia que los profesionistas urbanos sienten con los libros se asemeja a las narraciones orales que a través de cuentos y leyendas transmiten los abuelos a los jóvenes tsotsiles. La pregunta que pende en el aire parafraseando a FASHEH (2005) es entonces ¿cómo promover la literacidad en español sin erradicarla en tsotsil pues hay una clara afectividad de los jóvenes en relación a la lengua tsotsil?

\section{REFERENCIAS}

ALEJOS, Elixer Ixba. La creación del Libro de Texto Gratuito en México (1959) y su impacto en la industria editorial de su tiempo. RMIE, 2013, Volumen 18, número 59, páginas 1189-12011. ISNN 14056666. Disponible en http://www.scielo.org.mx/pdf/rmie/v18n59/v18n59a8.pdf. Accedido el 19 de mayo del 2019. CASSANY, Daniel; Castellá, Josep M. Aproximación a la literacidad crítica. Perspectiva, Florianópolis, v. 28, n. 2, 353-374, jul/dez.2010 Disponível em: https://periodicos.ufsc.br/index.php/perspectiva/article/view/2175-795X.2010v28n2p353.

Aceso em: 25 abril 2019.

ENGLAND, Nora C. Introducción a la Lingüística: Idiomas mayas. Guatemala: Editorial Cholsamaj/ Proyecto Lingüístico Francisco Marroquín., 1996.

FERREIRA, Emilia. Cultura escrita y educación. México: Fondo de Cultura Económica, 1999.

FASHEH, Munir. Como erradicar o analfabetismo sem erradicar os analfabetos? In: Educação como exercício de diversidade. Brasília: UNESCO, MEC, ANPEd, 2005.

HEATH S. B. "What no bedtime story means: narrative skills at home and school". In Alessandro Duranti. Linguistic Anthropology. A Reader. Second Edition. New Jersey: Blackwell Publishing, 2011.

INSTITUTO NACIONAL DE LENGUAS INDÍGENAS. Catálogo de las lenguas Indígenas nacionales: variantes lingüísticas de México con sus autodenominaciones y referencias geoestadísticas. México: INALI, 2010.

INSTITUTO NACIONAL DE LENGUAS INDÍGENAS. ¿Qué es el INALI? Disponível em: https://www.inali.gob.mx/es/institucional.html Aceso em:17 feb 2019.

LUCHESSI, Dante. Introdução: Língua e Sociedade Partidas. In Língua e Sociedade Partidas.

A polarização sociolinguística do Brasil. São Paulo: Contexto, 2015, pp. 11-43. 
MEEK, Margarete. En torno a la cultura escrita. México: Fondo de Cultura Económica, 2004.

VILLANUEVA, Rebeca Barriga. Una hidra de siete cabezas y más: la enseñanza del español en el siglo XX mexicano. In VILLANUEVA, Rebeca Barriga; BUTRAGUEÑO, Pedro Martín. Historia sociolingüística de México. Volumen 2. México: El Colegio de México, 2010, p. 1095-1194.

STREET, Brian V. Literacy in theory and practice. New York: Cambridge University Press, 1995. 